\title{
Laparoscopic Treatment of Liver Abscess by a Fishbone Impaction: A Case Report
}

\author{
Hanzhang Dong ${ }^{\mathrm{a}}$, Xi Liu ${ }^{\mathrm{a}, \mathrm{b}}$, Mingjian Luo ${ }^{\mathrm{a}}$, Shaobiao Ke ${ }^{\mathrm{a}}$, Jiulin Zhan ${ }^{\mathrm{a}}$, Zhiwei Li ${ }^{\mathrm{a}, \mathrm{c}}$
}

\begin{abstract}
Bacterial liver abscesses caused by ingested bodies are very uncommon. In the current report, a unique case of a 50-year-old man associated with diabetes mellitus who suffered from sepsis and a liver abscess because of the migration of a fishbone that lodged in the left hepatic lobe is presented. The diagnosis was confirmed by enhanced computed tomography examination of the upper abdomen. The patient subsequently underwent laparoscopic surgery, and was discharged on postoperative day 12 without any complications. The present study discusses the clinical features and treatment of this case.
\end{abstract}

Keywords: Foreign body; Liver abscess; Laparoscopic surgery; Sepsis; Diabetes mellitus

\section{Introduction}

Bacterial liver abscess caused by the transfixion of foreign bodies through the gastrointestinal tract is extremely rare [1]. Most of the ingested foreign bodies pass uneventfully through the gastrointestinal tract. Perforation of the gastrointestinal occurs in less than $1 \%$ of ingestion of a foreign body [2]. Here we report a rare case of successful laparoscopic treatment of a bacterial liver abscess associated with diabetes mellitus by a fishbone impaction.

\section{Case Report}

In January 2021, a 50-year-old man with a medical history of type 2 diabetes mellitus for about 5 years was referred to the

Manuscript submitted July 17, 2021, accepted August 17, 2021

Published online September 30, 2021

aDepartment of Hepatobiliary and Pancreatic Surgery, Kanghua Hospital, Dongguan 523080, Guangdong Province, China

bSecond Clinical Medical College of Guangzhou University of Chinese Medicine, Guangzhou 510006, China

${ }^{\mathrm{c} C o r r e s p o n d i n g ~ A u t h o r: ~ Z h i w e i ~ L i, ~ D e p a r t m e n t ~ o f ~ H e p a t o b i l i a r y ~ a n d ~ P a n c r e-~}$ atic Surgery, Kanghua Hospital, Dongguan 523080, Guangdong Province, China.Email: lizhiwei67@126.com

doi: https://doi.org/10.14740/jcs444 local hospital complaining of upper abdominal pain without nausea or vomiting for 1 day. After medical treatment, the upper abdominal pain was alleviated but did not completely disappear. Due to the increased abdominal pain after 3 days, the patient was referred to Kanghua Hospital for treatment.

The admission charge showed temperature of $38.2{ }^{\circ} \mathrm{C}$, heart rate of 112 , blood pressure of $72 / 49 \mathrm{~mm} \mathrm{Hg}$, and respiratory rate of 23 . Physical examination revealed that the abdomen was flat, the abdominal muscle was a little tense in the upper abdominal, abdominal tenderness was positive, and rebound tenderness was negative in the upper abdominal, and bowel sounds were three times every minute. Laboratory examination revealed that a white blood cell count was $13.31 \times$ $10^{9} / \mathrm{L}$ (normal range, $3.5-9.5 \times 10^{9} / \mathrm{L}$ ), the neutrophil percentage was $92 \%$ (normal range, $40-75 \%$ ), the lymphocyte count was $0.62 \times 10^{9} / \mathrm{L}$ (normal range, $1.1-3.2 \times 10^{9} / \mathrm{L}$ ), the hemoglobin (Hgb) levels was $105 \mathrm{~g} / \mathrm{L}$ (normal range, 130 $175 \mathrm{~g} / \mathrm{L}$ ), the platelet (PLT) count was $103 \times 10^{9} / \mathrm{L}$ (normal range, $125-350 \times 10^{9} / \mathrm{L}$ ), the C-reactive protein was above $200 \mathrm{mg} / \mathrm{L}$ (normal range, $0-10 \mathrm{mg} / \mathrm{L}$ ), the procalcitonin was $61.52 \mathrm{ng} / \mathrm{mL}$ (normal range, $0-0.046 \mathrm{ng} / \mathrm{mL}$ ), the aspartate aminotransferase (AST) was $272 \mathrm{U} / \mathrm{L}$ (normal range, 9 - 50 $\mathrm{U} / \mathrm{L}$ ), the alanine aminotransferase (ALT) was $171 \mathrm{U} / \mathrm{L}$ (normal range, 15 - $40 \mathrm{U} / \mathrm{L}$ ), the total bilirubin was $15.7 \mu \mathrm{mol} / \mathrm{L}$ (normal range, 0 - $26 \mu \mathrm{mol} / \mathrm{L}$ ), the direct bilirubin was 10.4 $\mu \mathrm{mol} / \mathrm{L}$ (normal range, 0 - $26 \mu \mathrm{mol} / \mathrm{L}$ ), the albumin was 24.1 $\mathrm{g} / \mathrm{L}$ (normal range, $40-55 \mathrm{~g} / \mathrm{L}$ ), and the blood sugar was 21.2 $\mathrm{mmol} / \mathrm{L}$ (normal range, $4.1-5.9 \mathrm{mmol} / \mathrm{L}$ ). The results of the abdominal ultrasonography revealed a mixed mass in the left hepatic lobe. Enhanced computed tomography (CT) examination of the upper abdomen revealed a needle-shaped dense shadow between the stomach and the liver (Fig. 1), a mass with ring enhancement $(8 \times 7 \mathrm{~cm})$ in the left hepatic lobe which was suspected to be a liver abscess (Fig. 2). The results suggested the patient suffered bacterial liver abscess caused by a foreign body. To inquest the case history, the patient denied eating any foreign body before abdominal pain. Subsequently, a gastric endoscopy was performed and nothing was revealed.

Blood glucose control was consulted by endocrinologists and then treated by insulin via a venous pump. Because the liquefaction of the liver abscesses was not evident in our case, conservative treatment rather than surgery drainage was chosen. Laparoscopic surgery was performed under general anesthesia when the imaging of $\mathrm{CT}$ reexamination showed liquefaction within the lesion after antibiotic therapy by intravenous drip for 4 days (ceftazidime) (Fig. 3). In the operation, we found the adhesions at the omentum, liver, duodenum, 


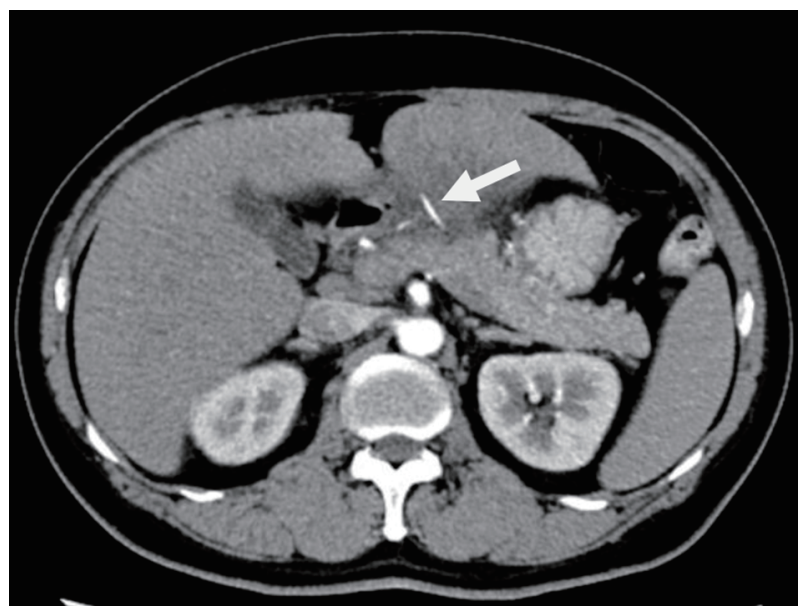

Figure 1. Enhanced computed tomography (CT) scan of the upper abdomen demonstrating the needle-shaped dense shadow (arrow) between the stomach and the liver.

and stomach, and the tissues around the left hepatic lobe had tightly adhered to the left liver. After carefully separating the adhesions around the left liver by ultrasonic scalpel, we found a foreign body in the omentum around the hepatoduodenal ligament and an abscess in the left hepatic lobe. Then the liver abscess was drained and the foreign body was removed (Figs. 4 and 5). Part of the pus was taken out for bacterial culture and drug sensitivity experiments.

There was approximately $200 \mathrm{~mL}$ white pus in the abscess cavity of the liver, and approximately $80 \mathrm{~mL}$ ascites in the abdominal cavity, mainly in the liver and kidney crypts. The foreign body was confirmed to be a needle-shaped fishbone with a length about $2.5 \mathrm{~cm}$ and a diameter of about $0.3 \mathrm{~cm}$ (Fig. 6). The abdominal local rinse was performed by warm saline and drainage pipes were placed in the abscess cavity of the liver and out of the abscess cavity of the liver, respectively (Fig. 7). Because blood cultures were positive for Streptococ-

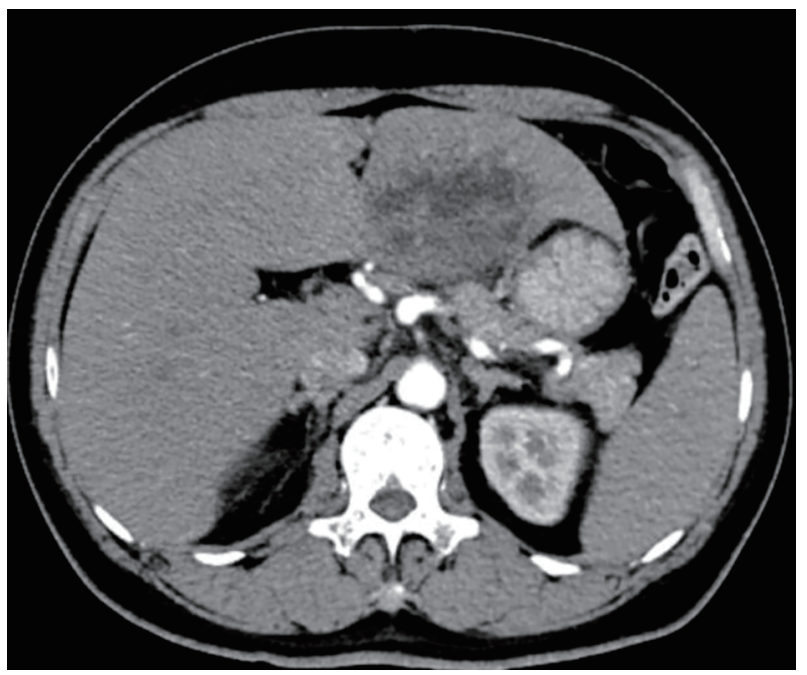

Figure 2. Enhanced computed tomography (CT) scan of the upper abdomen showing a $7 \mathrm{~cm}$ hypodense mass in the left hepatic lobe.

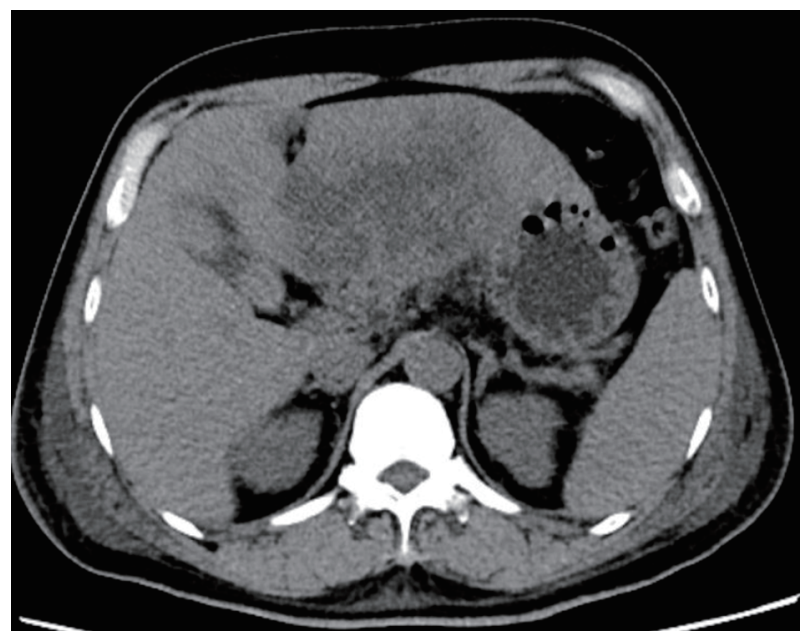

Figure 3. Plan computed tomography (CT) scan of the upper abdomen showing liquefaction within liver abscess after antibody therapy for 4 days before operation.

cus constellatus and drug sensitivity experiments resulted in sensitivity to imipenem cilastatin, the patient was treated with imipenem cilastatin by intravenous drip for 5 days after the operation. The drainage pipe out of the abscess cavity of the liver was removed on postoperative day 7 , and the drainage pipe in the abscess cavity of the liver was removed on postoperative day 12. The patient resumed oral intake on postoperative day 2 and was discharged on postoperative day 12 without any complications.

\section{Discussion}

Bacterial liver abscesses are a common disease. Generally, bacteria invade the liver through biliary tracts, hepatic arteries, or portal veins. Bacterial liver abscess caused by gastrointestinal

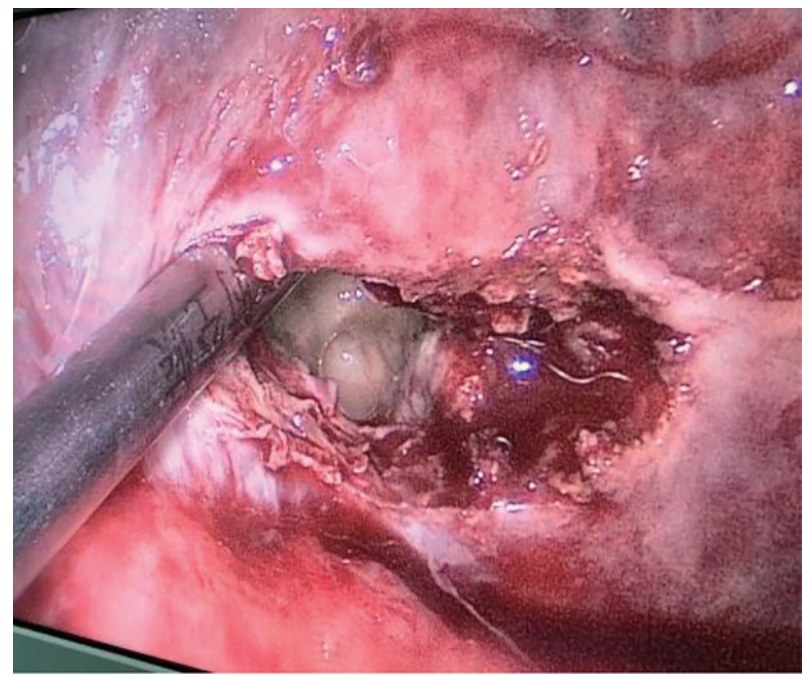

Figure 4. The hepatic abscess in the left hepatic lobe was opened by ultrasonic scalpel. 


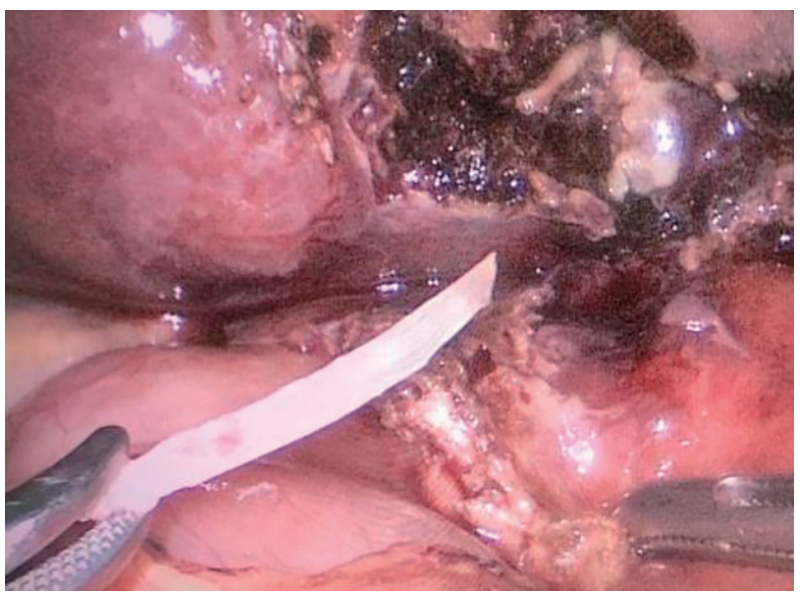

Figure 5. Removal of the fishbone in the omentulum around the hepatoduodenal ligament by forceps.

perforation of a foreign body is extremely rare. A fishbone is the most frequent foreign body accounting for approximately $40 \%$ of cases [3]. The left hepatic lobe was the most frequent site for bacterial liver abscesses caused by fishbones because of the proximity of the stomach to the left hepatic lobe [4]. In general, patients rarely recall the episode of the ingestion, and the foreign bodies may remain silent until they cause abscesses [5]. In the current case, the patient did not describe the ingestion of foreign bodies probably because the fishbone had been ingested a long time before the complication. We suspect that the fishbone had penetrated the stomach wall, stabbed into the left hepatic lobe and led to a bacterial abscess.

Most patients have non-specific symptoms and the preoperative diagnosis of bacterial liver abscess caused by foreign bodies can be challenging [6, 7]. As the delayed diagnosis of liver abscess caused by foreign bodies may lead to a lifethreatening infection, the complications should be comprehensively assessed by the medical history of the patient, laboratory examination, and imaging manifestation. Plain radiographic

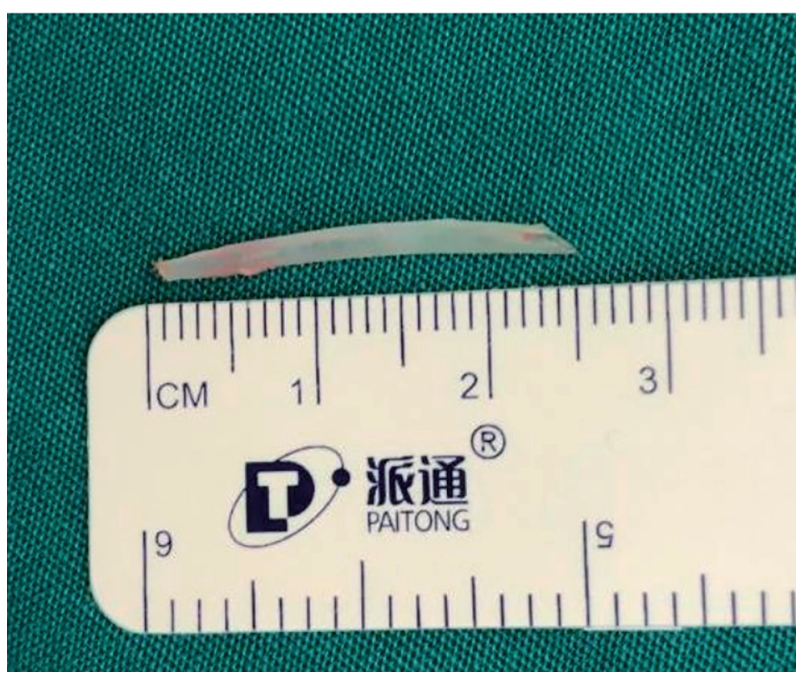

Figure 6. The foreign body removed: a $2.5 \mathrm{~cm}$ long fishbone.

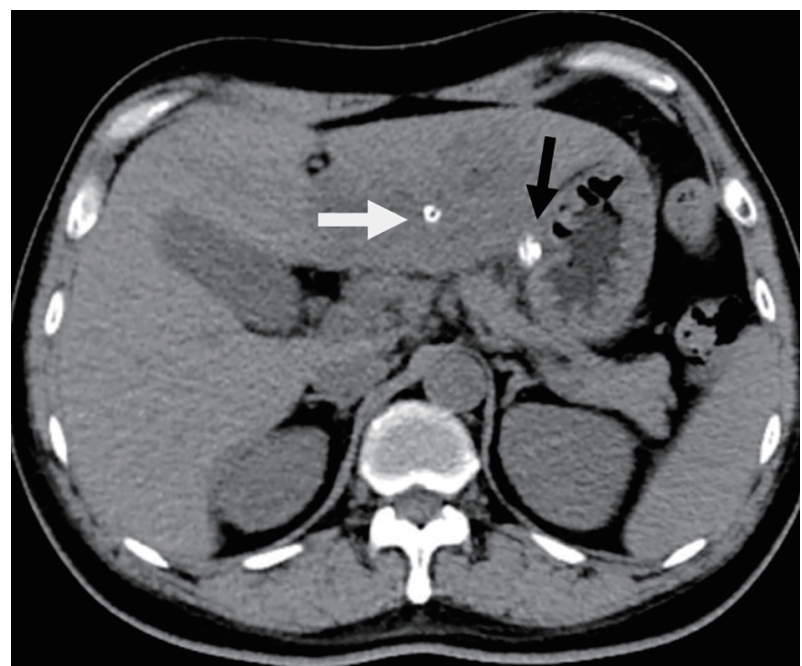

Figure 7. Plan computed tomography (CT) scan of the upper abdomen after operation showing the drainage pipe in the abscess cavity of liver (white arrow) and the drainage pipe out of the abscess cavity of the liver (black arrow).

tests are of limited assistance as many foreign bodies are not radiopaque or are too small to be seen $[8,9]$. Enhanced CT examination of the abdomen is the most important tool when there is suspicion for this situation $[10,11]$. Enhanced CT examination can also help to guide the selection of the therapeutic schedule $[12,13]$.

At present one of the most effective treatments for this situation is drainage of the hepatic abscess and removal of the foreign body by surgery. Compared with open surgery, laparoscopic surgery has advantages for small wounds, minimal postoperative pain, faster return to normal activation, a better cosmetic outcome, slight influence on the abdominal tissues, and shorter hospital stay $[14,15]$. In this case, we adopted the laparoscopic surgery for drainage of the hepatic abscess and removal of the fishbone. Inspection of the surgical specimen revealed a fishbone measuring $2.5 \mathrm{~cm}$ in length. It is clear that the patient had eaten fish before the abdominal pain.

For the abscess with poor liquefaction and multilocular abscess, the effect of early drainage is poor. Antibiotic treatment should be given first. With the destruction of the internal compartment of the abscess and the gradual liquefaction of the lesions, the effect of surgical drainage is good. Studies have shown that Klebsiella pneumonia has replaced Escherichia coli as the main pathogen of bacterial liver abscess in Asian populations [16, 17]. The results of blood bacterial culture and drug sensitivity experiments of this patient showed that Streptococcus constellatus was found and was sensitive to imipenem cilastatin. The bacterial culture of pus did not cultivate bacteria. This is not the same as the previous reports. Diabetics are the high-risk population of bacterial liver abscesses. For diabetics with bacterial liver abscess, ineffective blood glucose control can lead to a poor prognosis. The uncontrolled blood glucose in diabetics with bacterial liver abscesses is prone to complications such as pulmonary infections, which increases the mortality of patients. Blood glucose control should be under the guidance of endocrinologists. Bacterial liver abscesses 
caused by foreign bodies require multidisciplinary treatment. And a reasonable and personalized therapeutic schedule should be determined.

In conclusion, we report an unusual case of a patient associated with diabetes mellitus who underwent laparoscopic surgery for the migration of an ingested fishbone that caused a hepatic abscess and sepsis. For such patients, enhanced CT scans are the most sensitive imaging test for the localization of suspected foreign bodies, and early diagnosis and treatment are crucial to decrease the risk of this potentially lethal condition.

\section{Acknowledgments}

None to declare.

\section{Financial Disclosure}

None to declare.

\section{Conflict of Interest}

None to declare.

\section{Informed Consent}

We hereby confirm that informed consent was obtained from the patient for publication of the case.

\section{Author Contributions}

HD, XL and ZL contributed to the development of the presented technique concept, literature research and writing of manuscript. ML, SK and ZJ contributed to the editing and reviewing of the manuscript.

\section{Data Availability}

The authors declare that data supporting the findings of this study are available within the article.

\section{References}

1. Santos SA, Alberto SC, Cruz E, Pires E, Figueira T, Coimbra E, Estevez J, et al. Hepatic abscess induced by foreign body: case report and literature review. World J Gastroenterol. 2007;13(9):1466-1470.

2. Leong HK, Chan RK. Foreign bodies in the upper digestive tract. Singapore Med J. 1987;28(2):162-165.

3. Leggieri N, Marques-Vidal P, Cerwenka H, Denys A, Dorta G, Moutardier V, Raoult D. Migrated foreign body liver abscess: illustrative case report, systematic review, and proposed diagnostic algorithm. Medicine (Baltimore). 2010;89(2):85-95.

4. de la Vega M, Rivero JC, Ruiz L, Suarez S. A fish bone in the liver. Lancet. 2001;358(9286):982.

5. Kanazawa S, Ishigaki K, Miyake T, Ishida A, Tabuchi A, Tanemoto K, Tsunoda T. A granulomatous liver abscess which developed after a toothpick penetrated the gastrointestinal tract: report of a case. Surg Today. 2003;33(4):312-314.

6. Perez Saborido B, Bailon Cuadrado M, Velasco Lopez R. A liver abscess secondary to a toothpick: a rare complication of accidental foreign body ingestion. Rev Esp Enferm Dig. 2019;111(2):167-168.

7. Allam M, Pericleous S. Migrated fish bone induced liver abscess: medical management. Pan Afr Med J. 2020;36:140.

8. Aftab Z, Ali SM, Koliyadan S, Al-Kindi N. Foreign body in the liver: Case report and review of literature. Qatar Med J. 2015;2015(1):5.

9. Kuzmich S, Burke CJ, Harvey CJ, Kuzmich T, Andrews J, Reading N, Pathak S, et al. Perforation of gastrointestinal tract by poorly conspicuous ingested foreign bodies: radiological diagnosis. Br J Radiol. 2015;88(1050):20150086.

10. Yeh HY, Chao HC, Chen SY, Chen CC, Lai MW. Analysis of radiopaque gastrointestinal foreign bodies expelled by spontaneous passage in children: a 15-year single-center study. Front Pediatr. 2018;6:172.

11. Martin S, Petraszko AM, Tandon YK. A case of liver abscesses and porto-enteric fistula caused by an ingested toothpick: A review of the distinctive clinical and imaging features. Radiol Case Rep. 2020;15(3):273-276.

12. Pai K, Pillai S, Bhandarkar A, Anand A, Sabhahit H. Migrating Ingested Foreign Body of the Upper Aerodigestive Tract with Resultant Septic Shock: Case report and literature review. Sultan Qaboos Univ Med J. 2013;13(4):606610.

13. Matrella F, Lhuaire M, Piardi T, Dokmak S, Bruno O, Maestraggi Q, Kianmanesh R, et al. Liver hilar abscesses secondary to gastrointestinal perforation by ingested fish bones: surgical management of two cases. Hepatobiliary Surg Nutr. 2014;3(3):156-162.

14. Chen J, Wang C, Zhuo J, Wen X, Ling Q, Liu Z, Guo $\mathrm{H}$, et al. Laparoscopic management of enterohepatic migrated fish bone mimicking liver neoplasm: A case report and literature review. Medicine (Baltimore). 2019;98(11):e14705.

15. Bekki T, Fujikuni N, Tanabe K, Amano H, Noriyuki T, Nakahara M. Liver abscess caused by fish bone perforation of stomach wall treated by laparoscopic surgery: a case report. Surg Case Rep. 2019;5(1):79.

16. Qu TT, Zhou JC, Jiang Y, Shi KR, Li B, Shen P, Wei $Z Q$, et al. Clinical and microbiological characteristics of Klebsiella pneumoniae liver abscess in East China. BMC Infect Dis. 2015;15:161.

17. Ontanilla G, Herrera JM, Alcivar JM, Martin-Gutierrez G, Marquez C, Marquez JL. Liver abscess due to Klebsiella pneumoniae and its relation to colon lesions. Rev Esp Enferm Dig. 2015;107(1):51-52. 